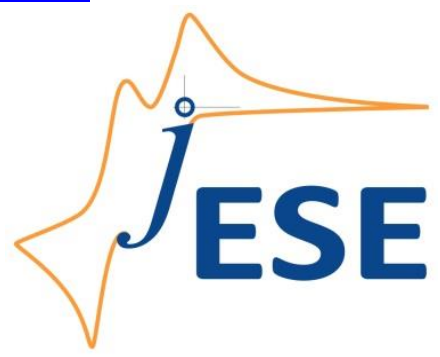

Open Access : : ISSN 1847-9286

www.jESE-online.org

Original scientific paper

\title{
Optical and electrochemical detection of a verotoxigenic $E$. coli gene using DNAzyme-labeled stem-loops
}

Gloria Longinotti ${ }^{1}$, Gabriel Ybarra ${ }^{1, 凶}$, Javier Montserrat ${ }^{2,3}$

${ }^{1}$ U.T. Nanomateriales, INTI-Procesos Superficiales, Instituto Nacional de Tecnología Industrial (INTI), Av. Gral. Paz 5445, B1650WAB San Martín, Argentina

${ }^{2}$ Universidad Nacional de General Sarmiento, J. M. Gutiérrez 1150, B1613GSX, Los Polvorines, Argentina

${ }^{3}$ Consejo Nacional de Investigaciones Científicas y Técnicas (CONICET), Argentina

Corresponding authors E-mail: ${ }^{\bowtie}$ E-mail: gabriel@inti.gov.ar; Tel./Fax: +51-11-4724-6333

Received: November 1, 2017; Revised: November 28, 2017; Accepted: November 30, 2017

\begin{abstract}
The activity of a peroxidase-mimicking DNAzyme was optimized to be used as a catalytic label in a stem-loop genosensor construction for quantifying the gene sequence Shiga-like toxin I of verotoxigenic E. coli. Experimental conditions such as $\mathrm{pH}$, buffer composition, potassium ion concentration, and hemin-to-oligonucleotides ratio, were analyzed to maximize optical and electrochemical responses using microvolumes. Different stem-loop constructions were evaluated to obtain the optimum response against the target concentration. Linear ranges of 0.05-0.5 $\mu \mathrm{M}$ and limits of detection of $174 \mathrm{nM}$ and 144 $n M$ were estimated for the optical and electrochemical measurements, respectively. Selectivity was proved by assaying other verotoxigenic, enterotoxigenic and enteroinvasive sequences. The results show that, if a combination of small-volume electrochemical cells and low-cost untreated screen-printed electrodes with a relatively high geometric area is used, electrochemical measurements present similar sensitivity and limits of detection to the more usual optical ones, allowing the development of lowcost electrochemical biosensors based on the use of soluble DNAzymes as labels.
\end{abstract}

\section{Keywords}

Biosensors; Genosensors; DNAzyme Peroxidase; Verotoxigenic E. coli

\section{Introduction}

Peroxidase-mimicking DNAzymes are complexes formed by oligonucleotides containing guanine quadruplexes and hemin as prosthetic group, which catalyze the decomposition of hydrogen peroxide with the consequent oxidation of a substrate. Since their first reports by Breaker [1] and Joyce [2] in the late 1990s, the possibility of using DNAzymes as artificial enzymatic labels in 
bioassays and biosensors has become apparent. DNAzymes have some advantages when compared to enzymes. DNAzymes are usually smaller than the corresponding enzymes, have a higher chemical stability, can be easily prepared using solid phase synthesis and can also be chemically modified. Moreover, their structures can be modulated for the adoption of active or inactive states. These versatile characteristics allow the combination, in a single DNA molecule, of both a recognition element and a catalytic label, as is the case of a peroxidase DNAzyme [3] combined with another DNA strand which acts as a biorecognition element [4]. Although this analytical strategy is attractive for the development of biosensors, its practical use can be hampered by a low sensitivity due to the lower catalytic activity of peroxidase DNAzymes when compared to peroxidase proteins.

The use of biorecognition-activated peroxidase-mimicking DNAzymes can be an especially attractive choice for the detection of complementary DNA sequences; for instance, those associated to bacterial toxins. In this regard, a considerable amount of work has been carried out for the detection of specific sequences of verotoxigenic $E$. coli (VTEC) or Shiga-toxigenic $E$. coli, including O157:H7 and other non-0157 serogroups, which produce verotoxins (verocytotoxins) that result in human diseases [5]. These toxins produce profound cytopathic effects in vero cells. Clinical symptoms may include bloody diarrhea and hemorrhagic colitis, along with complications associated with hemolytic-uremic syndrome (HUS), acute and chronic kidney disease, thrombotic thrombocytopenic purpura (TTP), neurological sequelae and death.

Optical detection has been the preferred means of transduction for the development of DNAzyme-based biosensors, usually with the use of ABTS, a reducing agent which becomes greenblue upon oxidation [4,6-9]. On the other hand, electrochemical transduction has been chosen when DNA strands were immobilized onto electroactive surfaces [10-12]. From the biosensors construction viewpoint, electrochemical detection shows a higher degree of electronic integration and a simplified design resulting from the fact that an electric signal is the natural result of electrochemical transduction [13]. Therefore, much effort has been devoted to developing optimized interactions between DNA strands and the electrode surface [14,15]. Although the use of soluble DNA structures avoids this issue, very few works have been published involving biosensors in which the catalytic activity of soluble DNAzymes is detected electrochemically [16].

Based on strategies similar to those reported previously [4-11,17], the aim of the present work was to prove the hypothesis that, by employing small-volume electrochemical cells in combination with inexpensive untreated printed electrodes, a sensitive detection of specific sequences of verotoxigenic $E$. coli could be carried out using DNAzymes as soluble electrochemical labels, yielding similar results to those obtained with the more usual optical detection. To that end, the conditions for the optimum response of a peroxidase DNAzyme were firstly optimized ( $\mathrm{pH}$, buffer composition, potassium ion concentration and hemin-to-oligonucleotides ratio) for electrochemical response. Secondly, different stem-loop oligonucleotides structures (recognition element and peroxidase DNAzyme) were designed and assayed for verotoxigenic $E$. coli sequences in order determine the best construction. Finally, response ranges, limits of detection and selectivity were studied for a stem-loop structure.

\section{Experimental}

\section{Reagents and preparations}

The sequences of DNA oligomers used are shown in Tables 1 and 2. The sequence showed in italic forms the DNAzyme known as $\mathrm{DZ5T}_{3}$ upon combination with hemin. In order to prepare the oligomer-hemin complex, the DNA oligomer stock solution was heated at $95{ }^{\circ} \mathrm{C}$ for 5 minutes and 
then diluted to a final concentration of $10 \mu \mathrm{M}$ with TKT buffer. The hemin stock solution in DMSO was diluted to a final concentration of $20 \mu \mathrm{M}$ with the same buffer. Finally, hemin was added to the oligomer solution with a hemin-to-DNA oligonucleotide ratio of 2:1. The mixture was allowed to stay at room temperature for 30 minutes.

Table 1. DNA oligomers used in the construction of the DZ5T 3 DNAzyme and stem-loop sequences against Shiga-like toxin I genes of verotoxigenic. Underlined: complementary sequences that form the stem.

Bold: $\mathrm{DZ5T}_{3}$ sequence. Italic: target recognition element against Shiga-like toxin I genes of verotoxigenic $\mathrm{E}$. coli

Sequence $\left(5^{\prime} \rightarrow 3^{\prime}\right)$

$\mathrm{DZ5T}_{3} \quad$ TTTGGGTAGGGCGGGTTGGG

Stem loop I TTTGGGTAGGGCGGGTTGGGTTTTTTAGAACGCCCACTGAGATCATCCAGTGTTGTCCCAACCC

Stem loop II TACCCAAAAGAACGCCACTGAGATCATCCAGTGTTGTTITTGGGTAGGGCGGGTTGGG

Stem loop III CCCAACCCAGAACGCCCACTGAGATCATCCAGTGTTGTTTTTGGGTAGGGCGGGTTGGG

Stem loop IV CCGCCCTAAGAACGCCCACTGAGATCATCCAGTGTTGTTTTTGGGTAGGGCGGGTTGGG

For the binding assay between the stem-loop and the target, the stock solutions of the DNA sequences were heated at $95{ }^{\circ} \mathrm{C}$ for $5 \mathrm{~min}$ and then diluted to a final concentration of $5 \mu \mathrm{M}$ with TKT buffer. Subsequently, different volumes of target sequence solutions were mixed with a volume of stem-loop solution in different tubes and brought to the final volume with TKT buffer. The mixture was heated at $57^{\circ} \mathrm{C}$ for 30 minutes. Finally, hemin was added and allowed to complex with DNA with stirring for 30 minutes at room temperature with a hemin-to-DNA ratio of 2:1. For the specificity assay, a similar procedure was followed employing the STXI sequence and five different oligonucleotides of others pathogenic $E$. coli (Table 2).

Table 2. Pathogenic E. coli sequences [18]

Sequence $\left(5^{\prime} \rightarrow \mathbf{3}^{\prime}\right)$

Shiga-like toxin I genes of verotoxigenic (STXI)

Shiga-like toxin II genes of verotoxigenic (STXII)

Heat-labile toxin genes of enterotoxigenic (IT)

Heat-stable toxin genes of enterotoxigenic (ST)

Adherence factor gene of enteropathogenic (EAF)

Invasiveness plasmid of enteroinvasive (IAL)
ACAACACTGGATGATCTCAGTGGGCGTTCT

GCGTTCTGTTCGCGCCGTGAATGAAG

AGCGGCGCAACATTTCAGGTCGAAGTCC

ATCAGAAAATATGAACAACACATTTTACTGCTGTGAAC

CGGCGCTGGTGATTTCGGGTTCGTCA

CTTATGTTCAAGGAAATAATTGTTGGCCTCCTTCTC

\section{Optical and electrochemical measurements}

UV-vis spectra were acquired with a Nanodrop 2000 Thermo Scientific spectrophotometer. Disposable acrylic cartridges with eight electrochemical cells were used for the electrochemical measurements $[19,20]$. Each cell with a volume of $40 \mu \mathrm{L}$ contained three screen printed electrodes: a carbon working electrode with an area of $1.6 \mathrm{~mm}^{2}$, a carbon counter electrode of approx. $10 \mathrm{~mm}^{2}$, and one $\mathrm{Ag} \mid \mathrm{AgCl}$ reference electrode. A Teq4 potentiostat was used.

\section{Results and discussion}

\subsection{Optical and electrochemical determination of the catalytic activity of DZ5T 3 -hemin DNAzyme}

Peroxidase-mimicking DNAzymes catalyze the reduction of hydrogen peroxide by ABTS, which is in turn oxidized to $\mathrm{ABTS}^{+\bullet}$. The rate of this reaction can be followed by measuring the absorbance at $420 \mathrm{~nm}$, the absorbance maximum of $\mathrm{ABTS}^{+\bullet}$ [21]. However, from the viewpoint of biosensors 
design, it is more convenient to perform a single measurement at a given time rather than to determine the reaction rate as a series of measurements. A higher sensitivity and a lower limit of detection could be obtained if the accumulated concentration of formed $\mathrm{ABTS}^{+\bullet}$ was determined after 3 minutes of reaction.

As reported earlier, the catalytic activity of peroxidase-mimicking DNAzyme depends on several factors, such as the concentration of potassium ions, buffer composition, $\mathrm{pH}$, and the ratio between the concentration of hemin and oligonucleotides [3]. In order to obtain a high sensitivity when DNAzymes are used as labels, the optimum conditions for the catalysis with DNAzymes must be established. Under the experimental conditions used, the concentration of potassium ions did not show a significant influence on the kinetics, although a minimum concentration of $1.3 \mathrm{mM}$ was found to be necessary. However, the hemin-to-oligonucleotide concentrations ratio and, especially, $\mathrm{pH}$ and buffer composition showed a strong influence on the reaction kinetics. The optimum values for the highest catalytic activity were found to be a pH of around 7.5 (Fig. 1a) and a ratio of hemin and oligonucleotides was found to be between 1.5 and 2.5 (Fig. 1b).
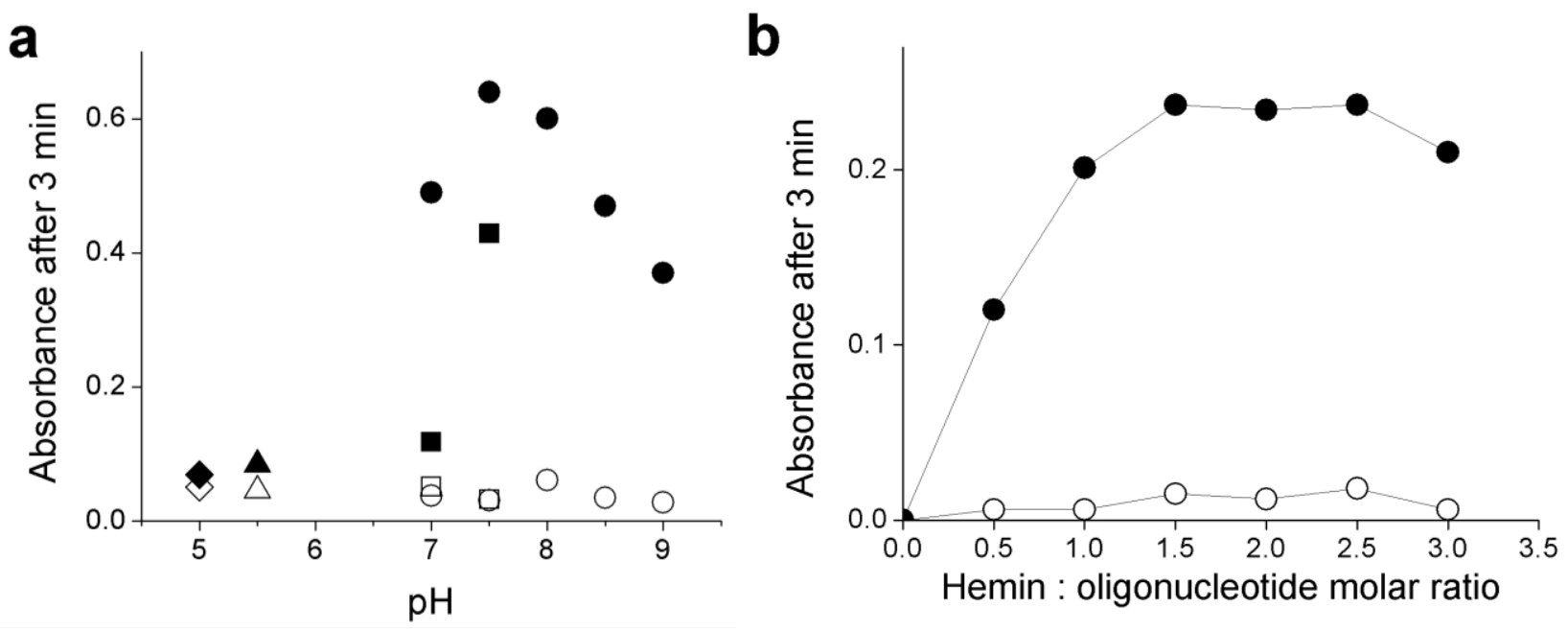

Fig. 1. (a) Dependence of the catalytic activity of $1 \mu \mathrm{M}$ DNAzyme (full) and $2 \mu \mathrm{M}$ hemin (empty) on $\mathrm{pH}$, determined by the absorbance measured at $420 \mathrm{~nm}$ developed by the oxidation of $50 \mu M$ ABTS after $3 \mathrm{~min}$ of reaction with $1 \mathrm{mM} \mathrm{H}_{2} \mathrm{O}_{2}$, oligonucleotide $0.31 \mu \mathrm{M}$, hemin $0.62 \mu \mathrm{M}, \mathrm{ABTS} 50 \mu \mathrm{M}$ in buffer $25 \mathrm{mM}$ : phthalate $(\bullet)$, acetate $(\mathbf{\Delta})$, phosphate $(\mathbf{\bullet})$, and tris- $\mathrm{HCl}(\bullet)$; (b) Dependence of the catalytic activity with hemin:oligonucleotide ratio, with $1 \mu M$ DNAzyme, 0.5-3 $\mu \mathrm{M}$ hemin, determined by the absorbance measured at $420 \mathrm{~nm}$ developed by the oxidation of $50 \mu \mathrm{M}$ ABTS after 3 min of reaction with $0.5 \mathrm{mM} \mathrm{H}_{2} \mathrm{O}_{2}$.

Electrochemical methods can also be used to determine the concentration of $\mathrm{ABTS}^{+\bullet}$. If a potential step is applied in conditions so that the limit current for the reduction of $\mathrm{ABTS}^{+\bullet}$ is attained, the limit current follows the well-known Cottrell equation [25]. Current transients (shown in Fig. 2a) followed a linear relationship between the limit current and the inverse of the square root of time, as expected from the Cottrell equation (inset in Fig. 2a). As can be seen in Fig. $2 b$, after a period of catalytic decomposition of hydrogen peroxide (in this case, $3 \mathrm{~min}$ ), the current measured at a given time after applying a potential step (20 s) was also directly proportional to the concentration of $\mathrm{DZ5T}_{3}$ DNAzyme. Therefore, soluble DNAzymes can also be used as labels whose activity can be determined by chronoamperometry. 
a

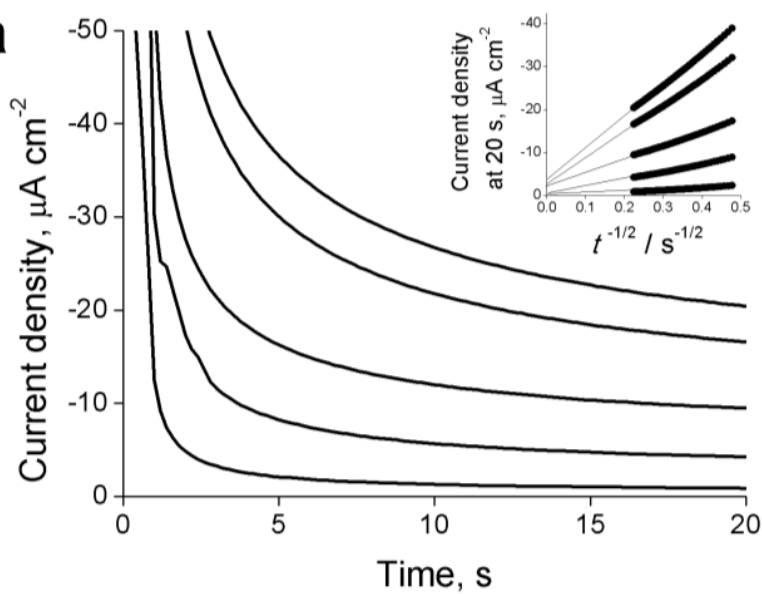

b

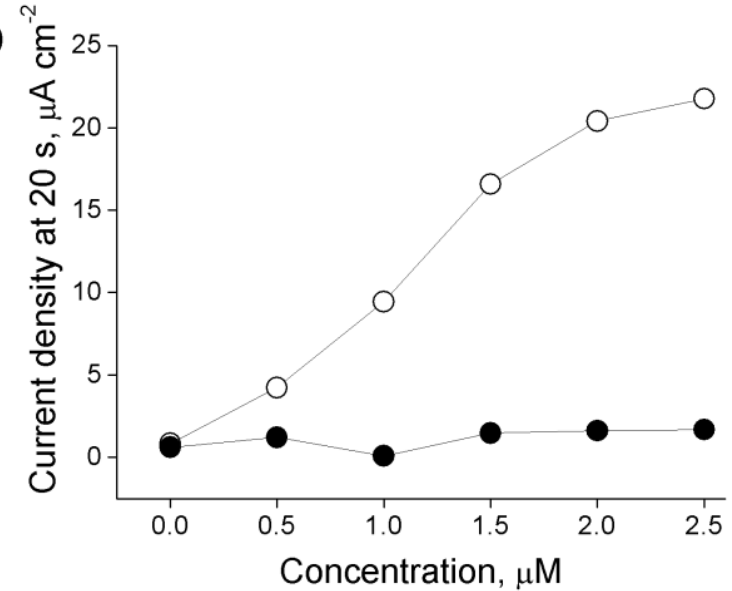

Fig. 2. (a) Current transients for different concentrations of DNAzyme, from bottom to top: 0-2.5 $\mu M$. Inset: plots of $\mathrm{i}$ vs. $\mathrm{t}_{\mathrm{m}}{ }^{-1 / 2}$; (b) Current measured at $20 \mathrm{~s}$ with a potentiostatic step after 5 min of reaction for different concentrations of DNAzyme (•) and hemin (0).

Optical and electrochemical determination of E. coli 0157 verotoxigenic gene using DNAzyme-labelled stem-loops

Four different DNA sequences (Table 1) differing in their complementary sequences were designed in order to analytically determine the presence of an E. coli 0157 gene. These constructions included the $\mathrm{DZ5T}_{3}$ sequence, a fragment which is complementary to a specific region of an E. coli verotoxigenic gen [18] and another moiety that allows the complete DNA sequence to be folded into a stem-loop structure. Fig. 3 shows the schematic representation for the detection of a target DNA strand with a stem-loop containing a complementary sequence and a DZ5T 3 sequence which adopts an active DNAzyme conformation after hybridization with the target.

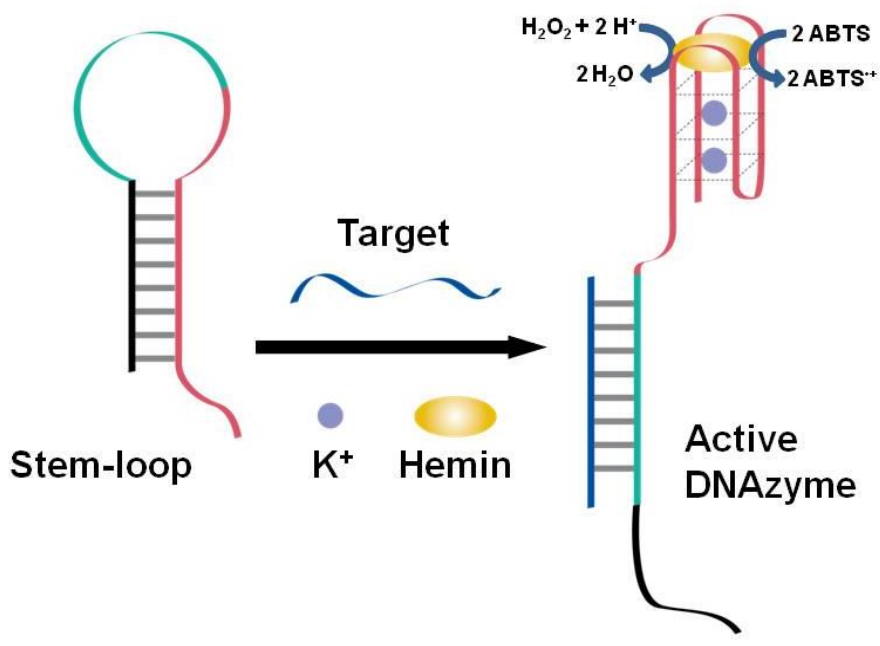

Fig. 3. Schematic representation of bioanalysis with a stem-loop for the recognition of a target DNA sequence upon activation of a DNAzyme. Black: complementary sequences to part of DZ5T 3 sequence. Green: complementary sequences to Shiga-like toxin I genes of verotoxigenic. Pink: DZ5T 3 sequence.

Blue: Shiga-like toxin I genes of verotoxigenic sequences

The activity of the four DNA stem-loops designed were spectrophotometrically tested (Fig. 4). In all cases, the sequence complementary to the target was located in the loop region and the stem length was 8 mer. The first construction (stem-loop I, Table 1) was designed with the $\mathrm{DZ5T}_{3}$ sequence located in the $5^{\prime}$ end, while the other constructions were prepared using the $\mathrm{DZFT}_{3}$ located in the $3^{\prime}$ end of the stem-loop. Another difference between the designs of the stem-loops was the 
moiety that blocked the $\mathrm{DZ5T}_{3}$ avoiding the active conformation. In the case of stem-loop I, the last 8 bases of the $3^{\prime}$ end of $\mathrm{DZ5T}_{3}$ were blocked. In all other three cases (stem-loops II-IV), different zones of the $\mathrm{DZ5T}_{3}$ sequence were explored.

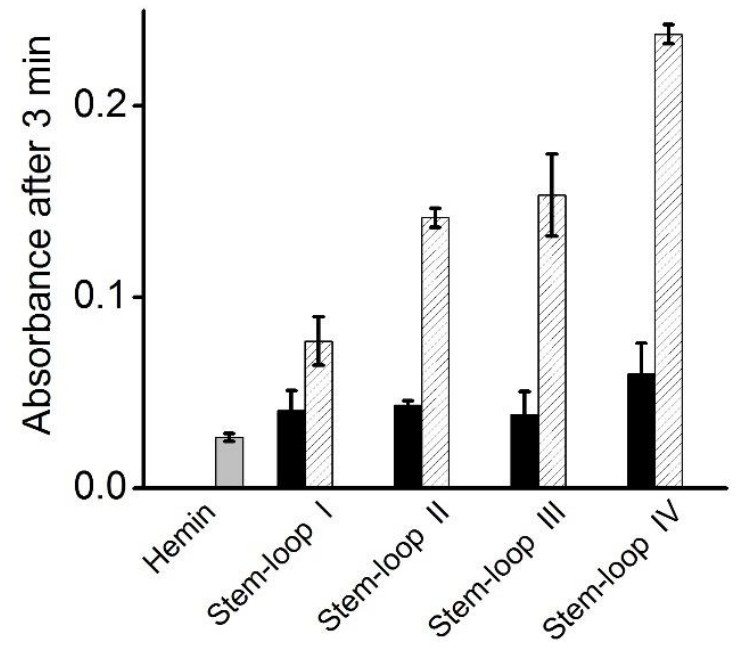

Fig. 4. Absorbance at $420 \mathrm{~nm}$ after 3 min of reaction for $1 \mu \mathrm{M}$ hemin (grey) and stem-loops I, II, III and IV $0.5 \mu \mathrm{M}+1 \mu \mathrm{M}$ hemin, in the absence (black) and the presence of $0.5 \mu \mathrm{M}$ target (strips).

A poor signal-to-noise ratio was obtained for stem-loop I. This low response could be related to an inappropriate stem-loop design. Zhang et al. [22] have reported that modifications at the 3' end of sequence corresponding to the peroxidase DNAzyme produced a decrease in the catalytic activity. It was assumed that, by adding bases at the $3^{\prime}$ end of the $\mathrm{DZ5T}_{3}$ oligonucleotide, the ability of the oligonucleotide to bind hemin decreases.

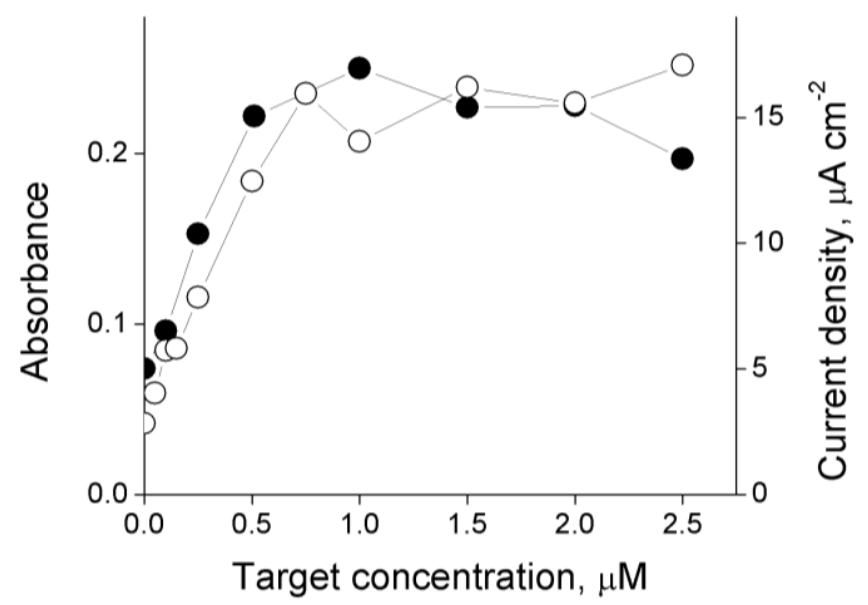

Fig. 5. Variation of absorbance $(\bullet)$ and limit current density at $20 \mathrm{~s}(0)$ after 3 min of reaction for a stemloop concentration of $0.5 \mu \mathrm{M}, 1 \mu \mathrm{M}$ hemine, $20 \mathrm{mM} \mathrm{KCl}, 0.2 \mathrm{M} \mathrm{NaCl}, 0.05 \%$ triton $x-100$, $3.4 \mathrm{mM}$ ABTS $0.5 \mathrm{mM} \mathrm{H}_{2} \mathrm{O}_{2}$.

For stem-loops II-IV, a significant increase in the absorbance with the concentration of the target was observed. These three constructions differ in the region where the $\mathrm{DZ}^{2} \mathrm{~T}_{3}$ sequence was blocked (near the $5^{\prime}$ end, the intermediate region, or near the $3^{\prime}$ end). No significant differences in the results were obtained with designs II and III. The results for stem-loop IV showed a higher sensitivity, and a maximum value of absorbance was obtained when stem-loop and target were used in a one-to-one ratio. Due to these results, stem-loop IV was chosen for successive measurements.

Fig. 5 shows the absorbance at $420 \mathrm{~nm}$ and the density current obtained at a fixed time ( $20 \mathrm{~s}$ ) produced by $\mathrm{ABTS}^{+\bullet}$ as a function of different concentrations of the target complementary sequence. 
It can be seen that the concentration of $\mathrm{ABTS}^{+\bullet}$ increases almost linearly for low values of target concentration and reaches a plateau for values higher than $1 \mu \mathrm{M}$. It can also be noted that even in the absence of the target sequence, a certain amount of $\mathrm{ABTS}^{+\bullet}$ is formed, which can be assumed to be the consequence of the formation of a certain amount of DNAzyme. From the data shown in Fig. 5 , it can be concluded that approximately $13 \%$ of the total possible concentration of available DNAzyme structure was formed in the absence of the target sequence. On the other hand, when an excess of target was used, the formation of the DNAzyme structure reached about $80 \%$ of the expected maximum value. For stem-loop IV, limits of detection of $174 \mathrm{nM}$ and $144 \mathrm{nM}$ were estimated for the optical and electrochemical measurements, respectively.

\section{Selectivity against Shiga-like toxin I verotoxigenic gene}

In order to evaluate the stem-loop IV selectivity for Shiga-like toxin I verotoxigenic gene (STXI, Table 2), an activity assay employing different sequences for other $E$. coli toxins was carried out. Fig. 6 shows the density current response for stem-loop IV $(1 \mu \mathrm{M})$ in the presence of different targets at the same concentration. Target sequences different from STXI showed responses similar to stem-loop IV in the absence of the STXI target, thus indicating the selectivity of the construction.

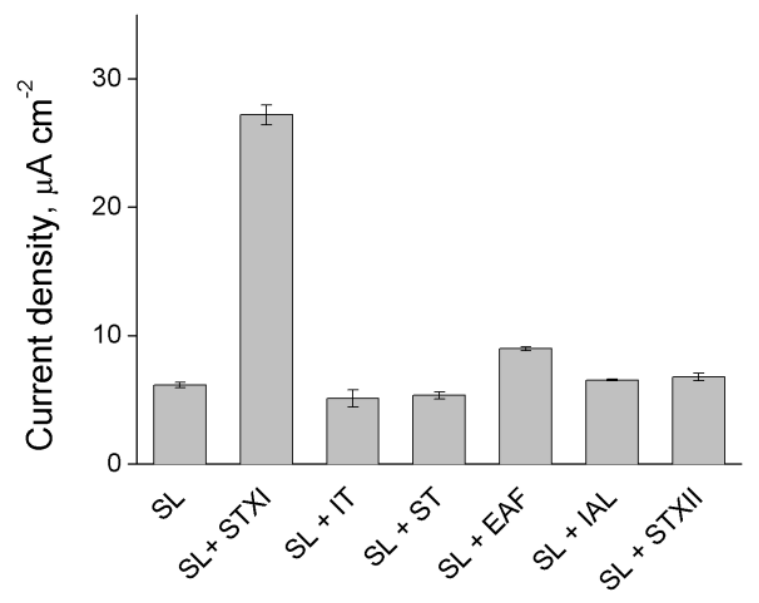

Fig. 6. Current density measured for the different E. coli pathogenic sequences (see Table 2) employing stem-loop IV.

\section{Conclusions}

The catalytic activity of a peroxidase-mimicking DNAzyme depends on factors such as $\mathrm{pH}$, buffer composition, potassium ions concentration, and the hemin-to-oligonucleotides ratio. For the $\mathrm{DZ}_{3} \mathrm{~T}_{3}$ DNAzyme, it was found that the buffer composition is as important as $\mathrm{pH}$ itself, and the highest catalytic activity was obtained when a TRIS buffer of $\mathrm{pH} 7.5$ was used. Additionally, a minimum amount of potassium ions of $1.3 \mathrm{mM}$ and a minimum hemin-to-oligonucleotides ratio of 2:1 were found to be necessary to achieve a high catalytic activity. DZ5T 3 DNAzyme was used as a label, combined in a stem-loop with a DNA sequence which recognizes a specific sequence of Shiga-like toxin I of verotoxigenic $E$. coli. It was demonstrated that the catalytic activity obtained was specific and proportional to the amount of the target concentration. The DZ5T 3 DNAzyme catalytic activity was followed optically and electrochemically. It is worth noting that soluble DNA species were used throughout this work, avoiding problems usually found with immobilized DNA strands, related to undesired DNA-surface interactions. The combination of electrochemical cells with relatively small volume and low-cost screen printed working electrodes with a relatively high geometric area allowed obtaining amperometric measurements with sensitivities and limits of detection 
comparable to optical methods. To our knowledge, this is the first report of a DNAzyme-based electrochemical bioanalysis with these features, which opens the way to the development of costeffective portable devices for the diagnosis of a verotoxigenic $E$. coli infection using of DNAzymes as labels.

\section{References}

[1] R. R. Breaker, Nature Biotechnology 16 (1997) 427-431.

[2] R. R. Breaker, G. F. Joyce, Chemical Biology 1 (1994) 223-229.

[3] P. Travascio, Y. Li, D. Sen, Chemical Biology 5 (1998) 505-517.

[4] Y. Xiao, V. Pavlov, T. Niazov, A. Dishon, M. Kotler, I. Willner, Journal of American Chemical Society 126 (2004) 7430-7431.

[5] D. L. Woodward, C. G. Clark, R. A. Caldeira, R. Ahmed, F. G. Rodgers, Canadian Journal of Infectious Diseases and Medical Microbiology 13 (2202) 321-330.

[6] P. Travascio, a J. Bennet, D. Y. Wang, D. Sen, Chemistry \& Biology 6 (1999) 779-787.

[7] D. Li, B. Shlyahovsky, J. Elbaz, I. Willner, Journal of American Chemical Society 129 (2007) 5804-5805.

[8] C. Teller, S. Shimron, I. Willner, Analitycal Chemistry 54 (2009) 9114-9119.

[9] J. Kosman, B. Juskowiak. Analytica Chimica Acta 707 (2011) 7-17.

[10] Q. Guo, Y. Bao, X. Yang, K. Wang, Q. Wang, Y. Tan, Talanta 83 (2010) 500-504.

[11] G. Pelossof, R. Tel-Vered, J. Elbaz, I. Willner, Analitycal Chemistry 82 (2010) 4396-4402.

[12] F. Li, L. Yang, M. Chen, Y. Qian, B. Tang, Biosensors \& Bioelectronics 41 (2013) 903-906.

[13] C. Moina, G. Ybarra, Advances in Immunoassay Technology, Norman H. L. Chiu, Ed. Zagreb, Croatia, 2012, pp. 65-80.

[14] F. Ricci, N. Zari, F. Caprio, S. Recine, A. Amine, D. Moscone, G. Palleschi, K. W. Plaxco, Bioelectrochemistry 76 (2009) 208-213.

[15] K. J. Cash, F. Ricci, K. W. Plaxco, Journal of American Chemical Society 131 (2009) 6955-6957.

[16] D. Zhang, J. Nie, F.-T. Zhang, L. Xu, Y.-L. Zhou, X.-X. Zhang, Analitycal Chemistry 85 (2013) 9378-9382.

[17] J. Kosman, Y. T. Wu, A. Gluszynska, B. Juskowiak, Analytical and Bioanalytical Chemistry 406 (2014) 7049-7057.

[18] L. Watterworth, E. Topp, H. Schraft, K. T. Leung, Journal of Microbiological Methods 60 (2005) 93-105.

[19] M. E. Cortina, L. J. Melli, M. Roberti, M. Mass, G. Longinotti, S. Tropea, P. Lloret, D. A. R. Serantes, F. Salomon, M. Lloret, A. J. Caillava, S. Restuccia, J. Altcheh, C. A. Buscaglia, L. Malatto, J. E. Ugalde, L. Fraigi, C. Moina, G. Ybarra, A. E. Ciocchini, D. J. Comerci, Biosensensors \& Bioelectronics 80 (2016) 2433.

[20] J. Molinari, C. Moina, G. Ybarra, Journal of Electrochemical Science and Engineering 5 (2015) 9-16.

[21] R. E. Childs, W. G. Bardsley, Biochemical Journal 145 (1975) 93-103.

[22] X. Zhou, M. Zhang, H. Li, M. Deng, X. Weng, H. Ma, S. Feng, Y. Zhou, Chemistry \&. Biodiversity 9 (2012) 170-180.

[23] F. Violante-Mota, E. Tellechea, J. F. Moran, G. Sarath, R. Arredondo-Peter, Phytochemistry 71 (2010) 21-26.

[24] A. J. Bard, L. R. Faulkner, Electrochemical Methods, Fundamentals and Applications, John Wiley \& Sons, U.S.A., 2001.

(C2017 by the authors; licensee IAPC, Zagreb, Croatia. This article is an open-access article distributed under the terms and conditions of the Creative Commons Attribution license

(http://creativecommons.org/licenses/by/4.0/) 